\title{
Multi-instrument observations of a large scale Pc4 pulsation
}

\author{
L. B. N. Clausen ${ }^{1}$, T. K. Yeoman ${ }^{1}$, R. Behlke ${ }^{2}$, and E. A. Lucek ${ }^{3}$ \\ ${ }^{1}$ Department of Physics and Astronomy, University of Leicester, Leicester LE1 7RH, UK \\ ${ }^{2}$ Department of Physics, University of Troms $\varnothing$, N-9037 Troms $\varnothing$, Norway \\ ${ }^{3}$ Department of Physics, Imperial College London, London SW7 2AZ, UK
}

Received: 20 June 2007 - Revised: 6 December 2007 - Accepted: 9 January 2008 - Published: 4 February 2008

\begin{abstract}
On 7 November 2005 various ground based and spaced based instruments registered five wave packets with frequencies in the Pc4 range. The most prominent of the five wave packets was observed in ground based magnetometer data spanning almost all latitudes on the dayside magnetosphere. The propagation from the dayside into the tail is deduced from Poynting flux calculations of Cluster data and an onset time analysis of the ground based magnetometer data. This suggests an upstream source. Backstreaming ions are identified to be the most probable source mechanism for this event. Due to the fortunate configuration of the Cluster satellites, the harmonic structure of the wave is analysed and compared with cross-phase spectra from ground data. We present evidence that the driving wave resonantly interacted with geomagnetic field lines. The data suggests that resonant driving occurred at stations where the driving frequency was harmonically related to the local fundamental frequency, creating FLR-like signatures.
\end{abstract}

Keywords. Magnetospheric physics (MHD waves and instabilities; Solar wind-magnetosphere interactions) - Space plasma physics (Waves and instabilities)

\section{Introduction}

In the dayside magnetosphere, ultra-low frequency (ULF) pulsations with spectral power in the Pc4 frequency range are abundant. The Pc4 interval ranges from 7 to $22 \mathrm{mHz}$ (periods between 45 and $150 \mathrm{~s}$ ) and the "Pc" reflects the fact that these pulsations tend to be continuous. They also occur less abundantly on the nightside.

Nightside Pc4 pulsations seem to be linked to substantial substorm activity, usually occurring two to four hours afterwards (e.g. Nosé, 1998). Particle data suggests that these pul-

Correspondence to: L. Clausen

(lbnc1@ion.le.ac.uk) sations are driven via a wave-particle interaction by freshly injected hot ions. Since most events occur during overall quiet geomagnetic times, a small convective electric field might also play a role. Through similar wave-particle interactions ions injected during substorms also drive Pc4 pulsations with high azimuthal wave numbers predominantly in the morning sector (Baddeley et al., 2002).

On the dayside, Pc4 pulsations are thought to originate from either Kelvin-Helmholtz instabilities on the magnetopause (Greenstadt et al., 1979) or wave-particle instabilities in the Earth's upstream region (Troitskaya et al., 1971; Le et al., 2000). An analysis of the occurrence region and propagation of the wave packets later in this paper will point towards the second mechanism, hence we can ignore the first mechanism in the context of this study.

When the orientation of the interplanetary magnetic field (IMF) is roughly (anti-)parallel to the normal vector of the bow shock, ions can be reflected at the boundary and travel upstream. Here they resonantly interact with naturally occurring waves, amplifying them (Barnes, 1970; Sentman et al., 1981). Since upstream the bow shock the solar wind is supersonic, these waves are then convected with the solar wind flow into the magnetosphere. Numerical hybrid models have been used to show that these fast/magnetosonic waves can then pass through the turbulent bow shock and magnetosheath into the dayside magnetosphere, leaving their spectrum mainly unchanged (Krauss-Varban, 1994).

Having reached the magnetosphere, compressional Pc3-4 waves are detected by space based instruments (e.g. Chi and Russell, 1998) and on the ground by magnetometers. On the ground they appear usually as large scale waves with low azimuthal wave numbers (Odera et al., 1991). Several empirical relations have been derived to predict the upstream generated frequency from IMF conditions (e.g. Le and Russell, 1996).

Ground based magnetometers show a peak in Pc4 activity at latitudes below $60^{\circ}$ (Samson and Rostoker, 1972).

Published by Copernicus Publications on behalf of the European Geosciences Union. 
Continuous pulsations at latitudes above $60^{\circ}$ are generally observed at lower frequencies between 2 and $7 \mathrm{mHz}$ (periods between 150 and 600 s) and these are termed Pc5 pulsations (e.g. Samson et al., 1992). Due to the lengths of the field lines at these latitudes the Pc5 range is favoured and Pc4 would only be expected if the density along the field lines was significantly lower than normal.

High latitude Pc5 pulsations often have distinctive characteristics and they have are known as field line resonances (FLR). Dungey proposed that they are standing Alfvén waves on geomagnetic field lines, a concept that has been confirmed by a number of studies (e.g. Walker et al., 1979). Since Chen and Hasegawa (1974) and Southwood (1974) have shown that energy from compressional wave modes can couple to Alfvénic modes, it is commonly accepted that compressional waves generated at either the Earth's bow shock or through solar wind buffeting can couple to FLRs where the frequencies match. Since the requirements are only met by a small fraction of field lines, FLRs tend to be latitudinally localized.

In this paper we discuss a highly coherent Pc4 pulsation with a $m$ number around unity. It is a global phenomenon, occurring at both low and high latitudes, over $12 \mathrm{~h}$ of magnetic local time (MLT). We explore the possibility of it being a FLR and investigate its source using ground and space based magnetometer data.

\section{Observations}

On 7 November 2005 between 13:10 and 14:10 UT over two dozen ground based magnetometers around the world registered five wave packets in the upper end of the Pc4 range. During this time, the magnetic foot prints of Cluster 3 and 4 of the four Cluster satellites (Escoubet et al., 2001) were, according to the Tsyganenko 96 (T96) model (Tsyganenko, 1995), conjunct with magnetometers belonging to the CARISMA array located in Canada. The CARISMA array spans magnetic latitudes from $60^{\circ}$ to $80^{\circ}$ and magnetic longitudes from $270^{\circ}$ to $330^{\circ}$. For the time discussed here, this translated to MLTs between 04:00 and 09:00, i.e. on the dawn flank of the magnetosphere. At the same time and nearly symmetric to the morning side stations, magnetometers belonging to the SAMNET and IMAGE arrays on the evening side between 14:00 and 17:00 MLT registered the same oscillations. These stations span magnetic latitudes from $50^{\circ}$ to $75^{\circ}$ at magnetic longitudes between $70^{\circ}$ and $120^{\circ}$.

\subsection{Ground based magnetometers}

Figure 1 shows the $\mathrm{X}$ (North-South) component of the magnetic field as measured by magnetometers along three latitude profiles at different MLTs in the left panels. The data have been bandpass filtered between 20 and $80 \mathrm{~s}$. The magnetic latitude of each station and its MLT is given next to its abbreviation in the top left hand corner of each panel. The right hand side panels show the Fourier spectrum of the filtered time series, each individually normalized. The spectra have been smoothed using a five points wide boxcar average to eliminate spikes.

As indicated in Fig. 1 by vertical dashed lines, five separate ULF wave packets can be identified at all stations. The first between 13:12 and 13:21 UT, the second between 13:23 and 13:27 UT, the third between 13:30 and 13:42 UT, the fourth between 13:43 and 13:48 UT and the fifth between 13:48 and 13:53 UT. The focus of this study lies on the wave packet which occurred between 13:30 and 13:42 UT. It was the most prominent of the five, already well defined in unfiltered data.

Basic Fourier analysis of the interval between 13:10 and 14:10 UT shows that the frequency of the first four pulsations was $17.2 \pm 1.0 \mathrm{mHz}$ whereas the last oscillated at $20.2 \pm 1.0 \mathrm{mHz}$ (see right panels in Fig. 1). The power at $20.2 \mathrm{mHz}$ dominates the power spectra of the northern IMAGE stations SOD, IVA, KEV, whereas in all other spectra the $17.2 \mathrm{mHz}$ peak is dominant. The frequency of each wave packet was constant over all latitudes. Although not immediately apparent from Fig. 1, cross-correlation techniques reveal that all five packets can in fact be seen in the data of all stations. The bandwidth of the filter in Fig. 1 was chosen such that any power at double the dominant frequency, i.e. at $34 \mathrm{mHz}$, is not filtered. There was, however, no signature present at this frequency.

The top six panels of Fig. 1 show the data of a line of magnetometers belonging to the CARISMA array in Canada spanning about $20^{\circ}$ in magnetic latitude, located around 07:00 MLT. The amplitude distribution of the third wave packet over latitude has two maxima, at ISLL and FCHU. Both phase and onset time vary with latitude, however the frequency does not. There is a distinct $180^{\circ}$ phase shift between the time series of GILL and FCHU but only a very small shift across the latitude of ISLL. Therefore the amplitude and phase profile over FCHU are indicative for the fact that the observed pulsation is at these stations is a FLR.

When studied in detail the pulsation between 13:30 and 13:42 UT consisted of two overlapping yet separate wave packets. SAMNET and IMAGE data (bottom panels in Fig. 1) show this more clearly than data from CARISMA stations. The further north the station, the further apart the two wave packets appeared. Since the pulsation occurred simultaneously at all stations, this indicates that it was a spatial feature rather than a temporal one.

The next seven panels in Fig. 1 show data from a latitude profile of magnetometer stations belonging to the SAMNET chain. The profile is located along $78^{\circ}$ magnetic longitude (14:30 MLT). A wave packet with the same frequency was observed between 13:30 and 13:42 UT, albeit with a smaller amplitude. Studying latitude profiles of spectral power for these stations at the observed frequency show two maxima in amplitude, one at HLL and the other at ESK. When only 

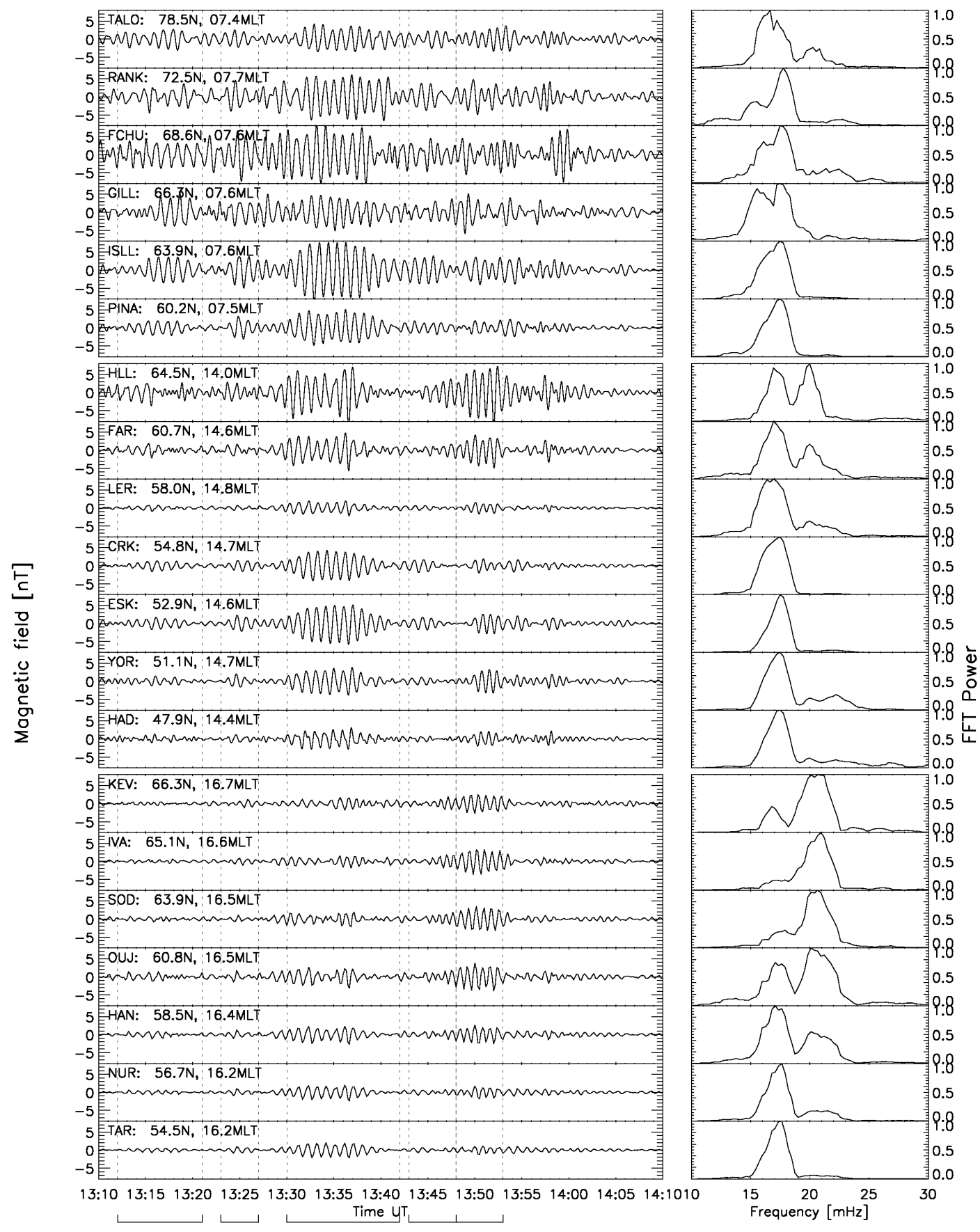

Fig. 1. Bandpass filtered (20 to $80 \mathrm{~s}$ ) ground based magnetometer data from three latitudinal profiles using CARISMA, SAMNET and IMAGE stations on the left. Five pulsation events are marked by vertical dashed lines. The panels on the right show the normalized smoothed Fourier spectra.

considering stations up to LER $\left(58^{\circ} \mathrm{N}\right)$, latitude profiles of phase and power show FLR-like behaviour.

At higher latitudes, the oscillation consisted of two packets, as seen in data from CARISMA stations. However, since SAMNET stations reach to lower latitudes, the merge was completed below $54^{\circ}(\mathrm{CRK})$ such that the oscillation then seemed to consist of only one wave packet.
Data of a latitude profile of stations belonging to the IMAGE chain are shown in the seven bottom panels of Fig. 1. These stations stretch along a line at $105^{\circ}$ magnetic longitude (16:00 MLT). Again, the same pulsations as in the two other chains were observed with even lower amplitudes. No signs indicating a FLR were observed in the spectral power and phase profiles. The splitting of one wave packet at lower 
Table 1. Phase in degree at $17.2 \mathrm{mHz}$ for different stations along longitudinal profiles determined by Fourier analysis.

\begin{tabular}{cccccc}
\hline \multicolumn{3}{c}{ Dawn } & \multicolumn{3}{c}{ Dusk } \\
\hline Name & Long. & Phase & Name & Long. & Phase \\
\hline DAWS $^{\ddagger}$ & 273 & -59 & & & \\
FSIM $^{\ddagger}$ & 294 & -37 & DOB $^{\star}$ & 090 & -40 \\
FSMI $^{\ddagger}$ & 306 & -24 & HAN $^{\star}$ & 104 & -47 \\
RABB $^{\ddagger}$ & 318 & -04 & MEK $^{\star}$ & 108 & -53 \\
Diff. & 45 & 55 & & 18 & -13 \\
& & & & & \\
$m$ & -1.2 & & & 0.7 \\
\hline
\end{tabular}

$\ddagger$ CARISMA stations

* IMAGE stations

latitudes into two at higher latitudes was again observed.

To determine the azimuthal wave number $m$ of the observed pulsation, phase shifts at stations along longitudinal profiles both on the dusk and dawn side have been analysed. The phase of the $17.2 \mathrm{mHz}$ component was obtained from Fourier analysis of the unfiltered signal. A summary is given in Table 1. The $m$ number is then obtained by dividing the observed phase shift by the longitudinal separation. By convention the eastward direction is associated with positive $m$ numbers.

With increasing longitude the phase increases on the morning side, corresponding to a negative $m$ number and westward propagation. On the evening side, the phase decreases with increasing longitude resulting in a positive $m$ number, equivalent to eastward propagation. This corresponds in both cases to a propagation of the wave in an anti-sunward direction. The absolute value is in both cases around one, confirming that this wave was a large-scale phenomenon.

Although it is not shown, it is worth noting that pulsation signatures identical to those described above were observed on the dayside by magnetometers located on Greenland, South America and Antarctica. These wave packets were clearly a global magnetospheric phenomenon with the exact same frequency at all stations. From these additional sources only the Antarctic stations yielded enough power for analysis, however due to the low power construction of these magnetometers, the time stamp associated with each measurement is uncertain by up to two minutes, thus rendering these data useless for timing analysis.

Weak but coherent signals of the pulsation described here were also recorded at stations located on the nightside. Data from Japanese magnetometers at magnetic latitudes between $20^{\circ}$ and $40^{\circ}$ and 23:00 MLT show pulsations at $17.2 \mathrm{mHz}$ with amplitudes around 0.2 nT. Magnetometers in Alaska $\left(60^{\circ}\right.$ magnetic latitude, 03:00 MLT) recorded the pulsation with an amplitude of $1 \mathrm{nT}$.
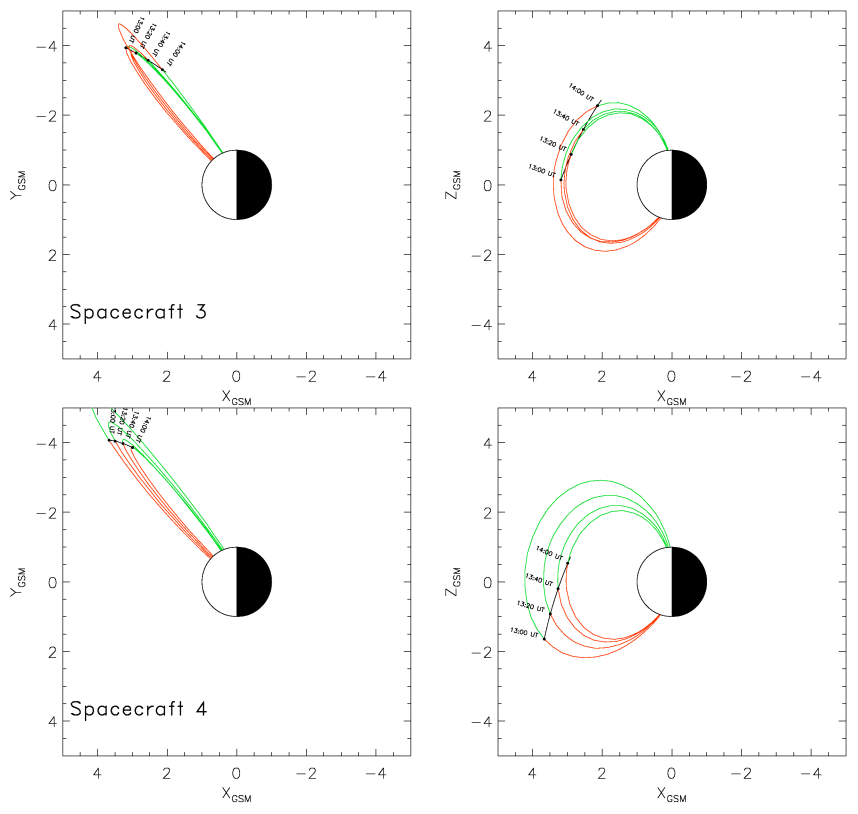

Fig. 2. Cluster 3 (top two panels) and 4 (bottom two panels) orbit. Also shown is the T96 model geomagnetic field with input parameters $p_{\mathrm{dyn}}=2.0 \mathrm{nPa}, D_{s t}=0.0, B_{y, \mathrm{IMF}}=0.0 \mathrm{nT}, B_{z, \mathrm{IMF}}=0.0 \mathrm{nT}$.

\subsection{Space based observations}

The formation of the Cluster satellites during the event was rather like pearls on a string. Cluster 2 was leading, followed by Cluster 1,3 and 4 , each separated by about $2 \mathrm{R}_{\mathrm{e}}$. Their orbit took them from the southern hemisphere through perigee at about $5 \mathrm{R}_{\mathrm{e}}$ over the northern hemisphere to apogee into the tail. Due to this orbit configuration, Cluster 3 and 4 sampled the magnetic and electric field variations along essentially the same bundle of field lines (see Fig. 2). At the time of the wave event Cluster 1 and 2 had already passed the inner magnetosphere and were on open field lines connected to the northern polar cap. Hence only Cluster 3 and 4 data can be used in this study.

The magnetic field was measured by the FGM (Balogh et al., 2001) instrument on board the Cluster satellites. It is available in GSE coordinates at spin resolution (4s). The electric field data used in this study was gathered by the EFW (Gustafsson et al., 1997) instrument. They were provided by the Cluster Active Archive and consist of two components in the GSE X-Y plane, also at spin resolution. The third component was calculated under the frozen flux assumption, i.e. $\boldsymbol{E}+\boldsymbol{v} \times \boldsymbol{B}=0$. It then follows that the electric and magnetic field ought to be orthogonal, i.e. $\boldsymbol{E} \cdot \boldsymbol{B}=0$ which yields

$E_{z}=-\left(E_{x} B_{x}+E_{y} B_{y}\right) / B_{z}$.

The results of the above calculation have to be treated very carefully whenever $B_{z}$ is close to zero. However, during this event the Cluster satellites were deep inside the magnetosphere close to the equatorial plane such that $B_{z}$ was 
of the order $200 \mathrm{nT}$, thus conveniently having avoided this problem.

After the $E_{z}$ component has been calculated from the raw magnetic and electric field data following Eq. (1), all components have been bandpass filtered to allow periods between 20 and $80 \mathrm{~s}$ and then transformed into a local dipole aligned coordinate system (LDC).

In this orthogonal system the background magnetic field is assumed to be purely dipolar. This assumptions is justified by the geocentric distance of the satellites of only $5 \mathrm{R}_{\mathrm{e}}$. One axis points along the background dipole magnetic field, such that positive values denote variations parallel to the local dipole field. The second axis points azimuthally, parallel to the X-Y GSM plane, positive eastward. The third axis completes the right hand system by pointing positive radially inwards. Thus, for magnetic field data the field aligned time series will contain the compressional component, the azimuthal direction will contain transverse toroidal oscillations and the radial component will contain the transverse poloidal oscillations.

The right panels in Fig. 3 show the Fourier spectra for the filtered time series. The spectral powers have been smoothed using a five point wide boxcar average to eliminate spikes. Then the spectra were normalized by the maximum value of each component for each quantity individually. Please note that the range of the $y$ axis is not the same for all spectra.

The left panels show time series of the magnetic and electric field and the oscillatory Poynting flux. All quantities are plotted versus time, L-value, MLT and magnetic latitude of the spacecraft. The dipole tilt angle respective to the Z-GSM axis during the event was about $-9^{\circ}$.

The top three left panels in Fig. 3 and Fig. 4 show the filtered components of the magnetic field for Cluster 3 and 4 in the LDC, respectively. All five wave packets are clearly visible in the data and the polarization of the wave is revealed. The variation at 17.2 and $20.2 \mathrm{mHz}$ was confined to the field aligned and azimuthal direction, showing that the wave consisted of a superposition of a transverse toroidal and a compressional mode. This is true for data from both Cluster 3 and 4.

The Fourier spectra show that the compressional and poloidal component of Cluster 3 magnetic field as well as the toroidal electric field contained a weak signal at double the main frequency, i.e. at $34 \mathrm{mHz}$. The same is true for the Cluster 4 data.

The envelopes of the main pulsation seen by the FGM on board Cluster 3 and 4 in the field aligned components show under close inspection two maxima, whereas the azimuthal components show only one.

The amplitude of the pulsation as seen by Cluster 3 was about twice that seen by Cluster 4 . Whereas the signal in the field aligned direction had no phase shift between the two spacecraft the oscillation in the azimuthal direction was $180^{\circ}$ out of phase.
The electric field shown in panels four to six in Fig. 3 and 4 also shows a clear polarization in the local dipole coordinate system. The bulk of the power was concentrated in the radial direction with all five wave packets clearly visible. The azimuthal component shows an elevated level of what seems to be incoherent noise. There was a coherent oscillation at $17.2 \mathrm{mHz}$ in the field aligned direction. The ratio of radial to field aligned amplitude was about ten at Cluster 3 and four at Cluster 4, making the field aligned component more significant at Cluster 4 . The signals from both satellites in the radial direction were in phase, as were the field aligned. A considerable difference in the packet's envelope was observed.

From the three components of the electric field $\boldsymbol{e}$ and the magnetic field $\boldsymbol{b}$ the three components of the Poynting vector $s$ have been calculated via

$\boldsymbol{s}=\frac{1}{\mu_{0}}(\boldsymbol{e} \times \boldsymbol{b})$.

Since $s$ was calculated from the filtered components it represents the Poynting flux of the wave field only.

Due to the polarization of $\boldsymbol{e}$ and $\boldsymbol{b}$ in the LDC the Poynting vector was polarized as well, having comparable amplitudes in the field aligned and azimuthal component (see Fig. 3 and Fig. 4 bottom three panels). The period of the oscillation was, as expected, double that of $\boldsymbol{e}$ and $\boldsymbol{b}$. The field aligned component of $\boldsymbol{s}$ for Cluster 3 showed a slight asymmetry around the zero line, indicating more flux parallel to the background magnetic field than anti-parallel. Since Cluster 3 was located about $12^{\circ}$ above the magnetic equator, this indicates that some of the wave's energy was lost in the northern polar ionosphere. The same is true for observations at Cluster 4 position, however here the energy was lost in the negative field aligned direction into the southern hemisphere, in accordance with the satellites position at $-10^{\circ}$ magnetic latitude.

The azimuthal flux at both satellites was, considering their position in the magnetosphere, purely tailward, i.e. westward, negative azimuthally. The asymmetries in both the field aligned/azimuthal flux is a direct and sole result of phase shift between the azimuthal/field aligned magnetic and radial electric field components at both satellites.

The small signals in the field aligned direction of the electric field results in some flux in a radially inward direction, more so at Cluster 4.

\section{Discussion}

\subsection{Wave motion and origin}

The observed pulsations were clearly a global phenomenon since they were observed by ground based magnetometers in different hemispheres spanning 12 hours of MLT. It is thus of special interest to discuss the structure and frequency of the wave in order to assess where it was generated and what the source mechanism was. Later, the configuration of the 


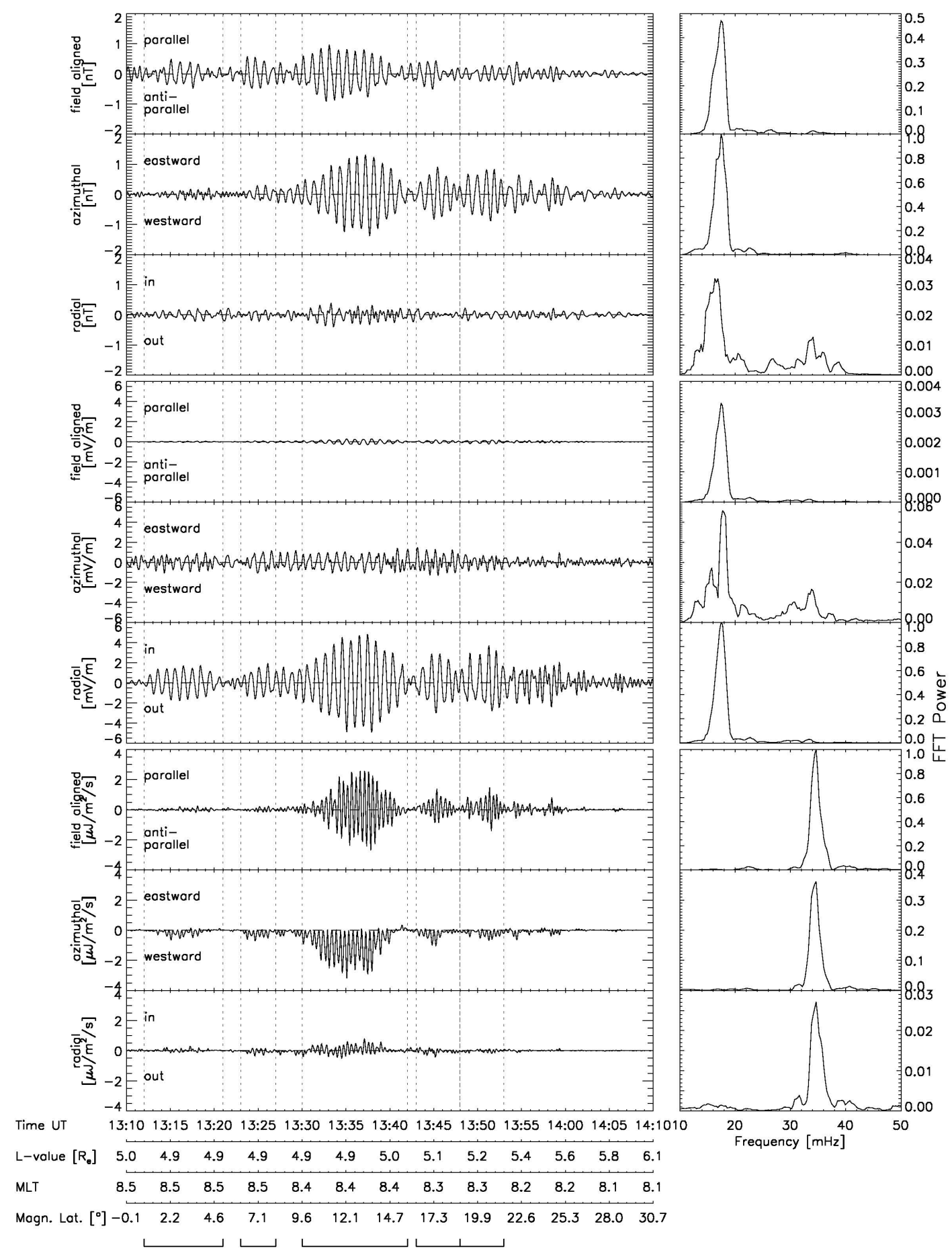

Fig. 3. Summary of field data in local dipole coordinates from Cluster 3 . The top three panels show the magnetic field, followed by the electric field and the oscillatory Poynting flux. The right hand side panels show the respective smoothed Fourier spectra.

Cluster tetrahedron gives us the unique opportunity to study the structure of the wave along a bundle of field lines and thus explore the wave's harmonic mode.

As discussed earlier, the magnetic field and Poynting flux data suggests that the wave was composed of two modes,
Alfvénic and compressional. While the Alfvénic flux oscillated along the background magnetic field between the two hemispheres depositing energy preferentially in the closer polar ionosphere, the flux of the compressional component (magnetic oscillation field aligned, electric oscillation radial) 


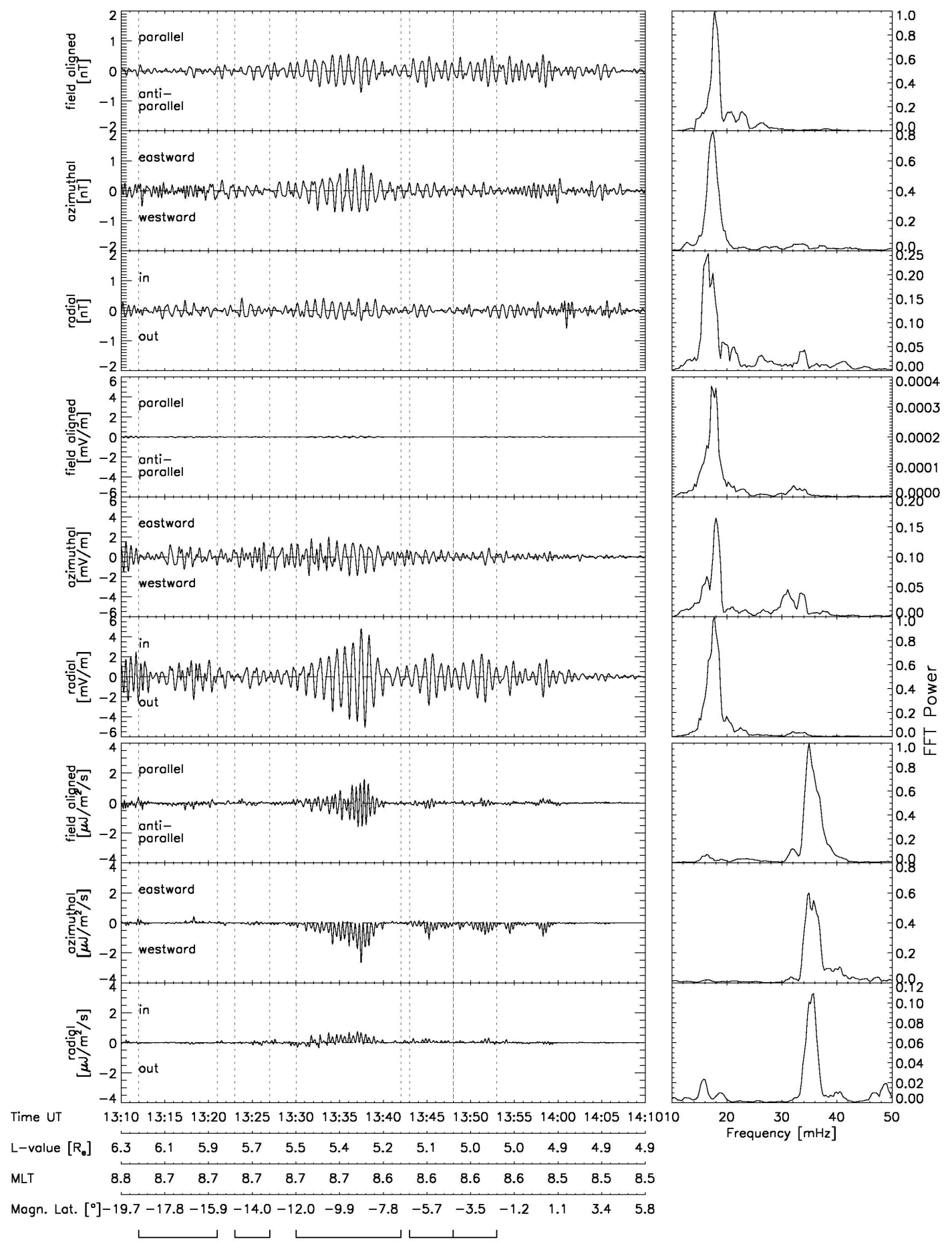

Fig. 4. Summary of field data from Cluster 4, same layout as Fig. 3.

was also highly asymmetric. Virtually all flux was directed tailward. This leads to the assumption of an anti-sunward travelling compressional wave from which energy was locally converted into the Alfvénic mode. The pulsations seen by the ground based magnetometers are then due to these Alfvénic oscillations.
Since the speed of the proposed compressional disturbance is finite, the onsets of the pulsations in the ground based measurements are dependent on this speed and on the position of the point where the adjacent field line of each station crosses the X-Y GSM plane. For convenience, this point will be called the cross-point in the following. 


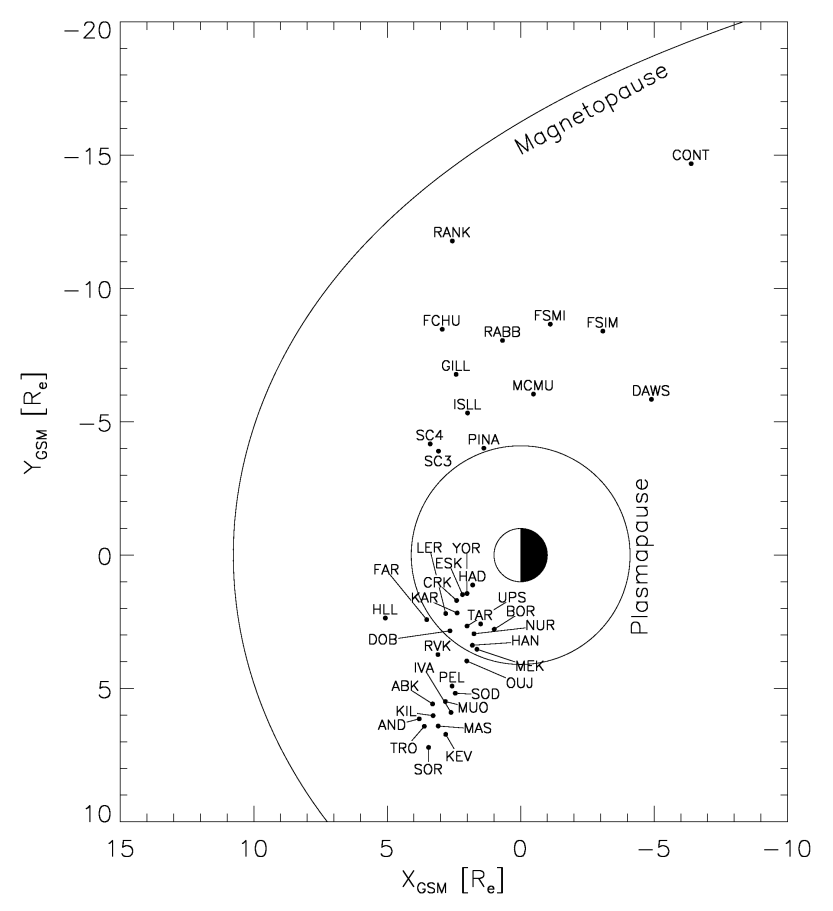

Fig. 5. Equatorial crossing points of field lines adjacent to ground magnetometer stations in GSM coordinates. The magnetopause position was calculated according to Shue et al. (1997). The plasmapause is shown following Larsen et al. (2007).

The geographic positions of each magnetometer belonging to the CARISMA, IMAGE and SAMNET chain have been traced along the adjacent field line into the equatorial plane of the GSM coordinate system (see Fig. 5), thus finding each station's cross-point. The tracing utilised the T96 model with input parameters derived from measurements of the GEOTAIL satellite $\left(p_{\text {dyn }}=1.5 \mathrm{nPa}, D_{s t}=-15.0, B_{y, \mathrm{IMF}}=-1.5\right.$ $\left.\mathrm{nT}, B_{z, \mathrm{IMF}}=-0.5 \mathrm{nT}\right)$. Due to the finite step size of the T96 model, the $\mathrm{Z}$ values of the equatorial positions were not zero, however they were less than $0.2 \mathrm{R}_{\mathrm{e}}$ for all stations.

To determine the onset time of at each station, the filtered time series between 13:30 and 13:42 UT of each station of the three chains have been cross-correlated with the time series of one reference station. HAD from the SAMNET chain was chosen to be the reference since it is the station in which time series the pulsation occurred first.

The sample interval of IMAGE stations is ten seconds as opposed to one second for SAMNET stations, therefore before cross-correlation the reference data have been downsampled by calculating the average over ten seconds. The same procedure was applied before cross-correlating the reference data with the four second sampled Cluster magnetic field measurements. Finally the onset time was then determined by extracting the lag of the maximum cross-correlation.

Tamao (1964) investigated the propagation of a MHD wave from a point source through a cold uniform plasma.

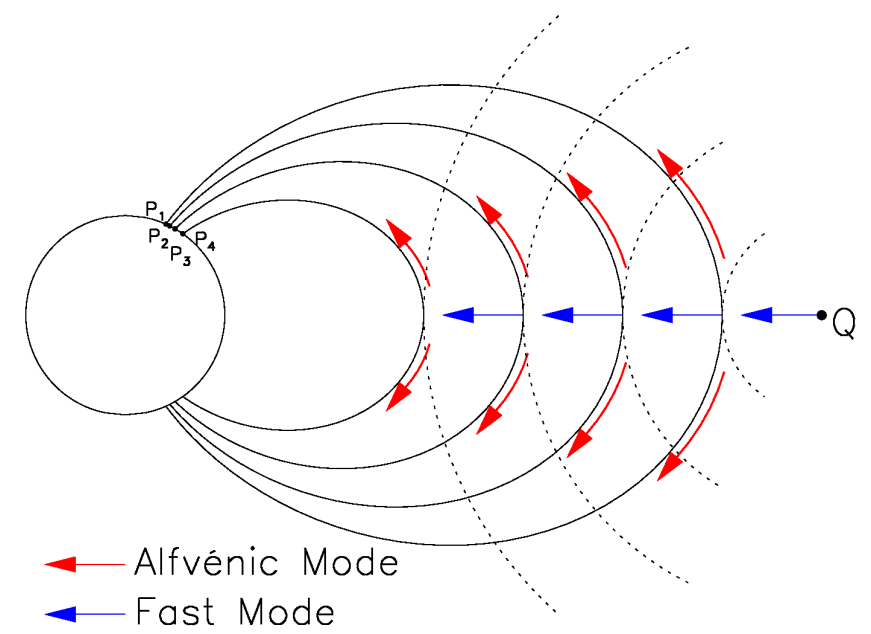

Fig. 6. MHD wave propagation inside the Earth's magnetosphere from a source $\mathrm{Q}$ to an observer $P_{1}, P_{2}, P_{3}, P_{4}$ on the ground. The path shown is that identified by Tamao (1964) as the least attenuated one.

He then applied his findings qualitatively to a MHD wave propagating through the magnetosphere to an observer on the ground.

The source Q emits compressional waves in all directions, as indicated by the dotted concentric lines in Fig. 6. These waves travel with the local Alfvén speed as the plasma is assumed to be cold, and they are attenuated as $r^{-1}, r$ being the distance from the source. Along its path the fast mode constantly converts to the Alfvén mode, which then travels along the magnetic field line without further attenuation to $P_{1}, P_{2}$, $P_{3}, P_{4}$, the observer on the ground. Hence most power is transported to the ground by a path which minimised the length the wave travels in fast mode. This path is depicted in Fig. 6 as a straight arrow in blue from the source to the field line connected to $P_{1}, P_{2}, P_{3}, P_{4}$, followed by the red arrow indicating the Alfvén mode travelling along the magnetic field line. This method was successfully used by Chi et al. (2001) to explain arrival times of preliminary reverse impulses.

Additional to the lag time due to different paths of the wave to the observer on the ground, the phase shifts resulting from an internal resonance structure of the pulsation will influence the observed onset times. Whereas the wave propagation along different paths will constitute the bulk of the lag time, the internal resonance structure of the pulsation can only change the lag times by \pm 30 s, i.e. \pm one half period.

In order to be able to estimate the travel times of the waves along the "Tamao path", the local Alfvén speed is needed. Here the T96 model provides the magnetic field for the calculations, the mass density $\rho(r)$ can be modelled in two parts, the first applying to the plasmasphere (subscript $p s$ ), the second to the magnetosphere (subscript $m s$ ) (compare Chi et al., 
2001)

$\rho(r)= \begin{cases}\rho_{p s}\left(\frac{r_{p p}}{r}\right)^{3}, & r \leq r_{p p} \\ \rho_{m s}\left(\frac{r_{m p}}{r}\right)^{3}, & r>r_{p p}\end{cases}$

For each station in Fig. 5 the travel time along a straight line from the source to the cross-point was calculated using Eq. (3) to model the density profile. The travel time of the Alfvénic mode along the magnetic field line as predicted by the T96 model was than added. Finally, the minimum travel time of all stations was subtracted from all travel times such that these relative times can be compared to the relative times determined by the afore mentioned cross-correlation technique.

Optimal values for the mass densities were found by systematically varying these parameters until the best agreement between observation and model was reached. $\rho_{p s}$ was varied between $25 \mathrm{amu} / \mathrm{cm}^{3}$ and $300 \mathrm{amu} / \mathrm{cm}^{3}$ in steps of $25 \mathrm{amu} / \mathrm{cm}^{3}$. The magnetospheric density was varied between $0.25 \mathrm{amu} / \mathrm{cm}^{3}$ and $2.0 \mathrm{amu} / \mathrm{cm}^{3}$ in $0.25 \mathrm{amu} / \mathrm{cm}^{3}$ steps. For each pair of density values model travel times were computed. The standard deviation of the differences between model and observational time lags of all stations has been used as a quantifier for the goodness of fit of the input density values. The densities producing the smallest deviation were then chosen as the optimal input parameters for our model. These values were $\rho_{m s}=0.75 \mathrm{amu} / \mathrm{cm}^{3}$ and $\rho_{p s}=50 \mathrm{amu} / \mathrm{cm}^{3}$ which achieved a minimal standard deviation of $7.8 \mathrm{~s}$.

A plasmaspheric density of $\rho_{p s}=50 \mathrm{amu} / \mathrm{cm}^{3}$ seems rather low, however note that changing this value to a more sensible value of $150 \mathrm{amu} / \mathrm{cm}^{3}$ increases the standard deviation marginally from 7.8 to $8.4 \mathrm{~s}$. On the other hand changing the magnetospheric density from 0.75 to $1.5 \mathrm{amu} / \mathrm{cm}^{3}$ increases the standard deviation to over $12 \mathrm{~s}$.

As mentioned earlier, the observational lag times of some stations might have been affected by additional time shifts due to local resonance effects. However the spread of the station's positions over large parts of the dayside of the magnetosphere both in MLT and L-shell (compare Fig. 5) means that only few of the stations will have been affected by such additional shifts. Hence this intuitive measure for the goodness of the model is suitable for this situation.

The geocentric distance of the plasmapause was derived from a recent model by Larsen et al. (2007). They found that the following parameters strongly correlate with the plasmapause position: the IMF Z-GSM component $B_{z}$, IMF clock angle $\theta=\arccos \left(B_{z} / \sqrt{B_{y}^{2}+B_{z}^{2}}\right)$ and a merging proxy $\phi=V B \sin (\theta / 2)^{2}$, where $V$ is the solar wind speed and $B$ is the IMF magnitude. The delay in the plasmapause response to the changing parameters was found to be $180 \mathrm{~min}$ for $B_{z}$, $175 \min$ for $\theta$ and $240 \mathrm{~min}$ for $\phi$. According to Larsen et al. (2007) the plasmapause position $L_{p p}$ can then be calculated via
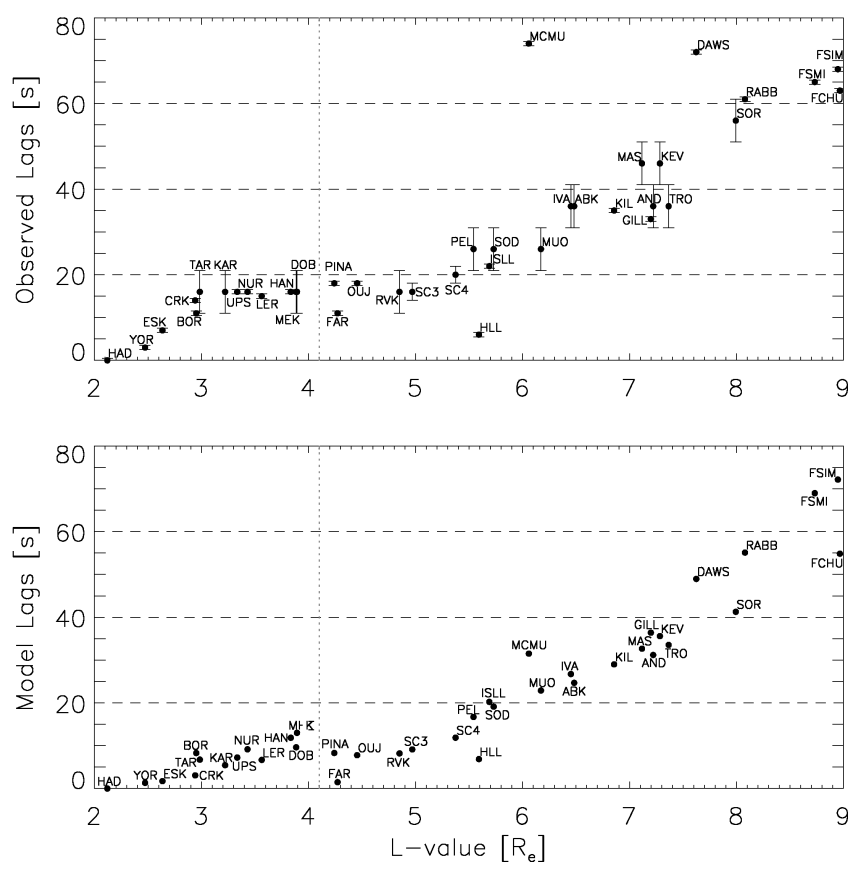

Fig. 7. Observed lag times (top panel) and relative predicted lag times (bottom panel). The dotted vertical line indicates the modelled plasmapause stand-off distance. The error bars in the top panel are due to the different sampling frequencies of the different magnetometers.

$$
\begin{array}{r}
L_{p p}\left[\mathrm{R}_{\mathrm{e}}\right]=0.050 B_{z, 180}+0.108 \theta_{175} \\
-1.110 \times 10^{-4} \phi_{240}+4.23,
\end{array}
$$

which in this case yields $r_{p p}=4.1 \mathrm{R}_{\mathrm{e}}$. Please note that this model does not account for the MLT dependence of $L_{p p}$.

For our calculations the magnetopause position was assumed to lie at $r_{m p}=10 \mathrm{R}_{\mathrm{e}}$. To find the location of the upstream source its position was varied using the same scheme as for the mass densities. For 80 points along a line parallel to the Y-GSM axis at $\mathrm{Z}_{\mathrm{GSM}}=0 \mathrm{R}_{\mathrm{e}}$ and $\mathrm{X}_{\mathrm{GSM}}=10 \mathrm{R}_{\mathrm{e}}$ between $-4 R_{e} \leq Y_{G S M} \leq 4 R_{e}$ the model lag time and the standard deviation of the difference between the lag times were calculated. It was found that the best agreement, i.e. the lowest standard deviation, between observation and model is achieved when assuming the source to lie at $(10,0,0) R_{e}$. This position is consistent with what will be inferred about the wave's origin later in this section.

The observed and the modelled lag times as a function of the L-value of each station are shown in Fig. 7. The general agreement between the predicted and observed travel times is good. The model predicts the minimum travel time for HAD station, a result which is also obtained by the crosscorrelation technique. Generally the modelled travel times are lower than those observed. Qualitative differences like "The pulsation first reached FAR, then OUJ and then PINA" 


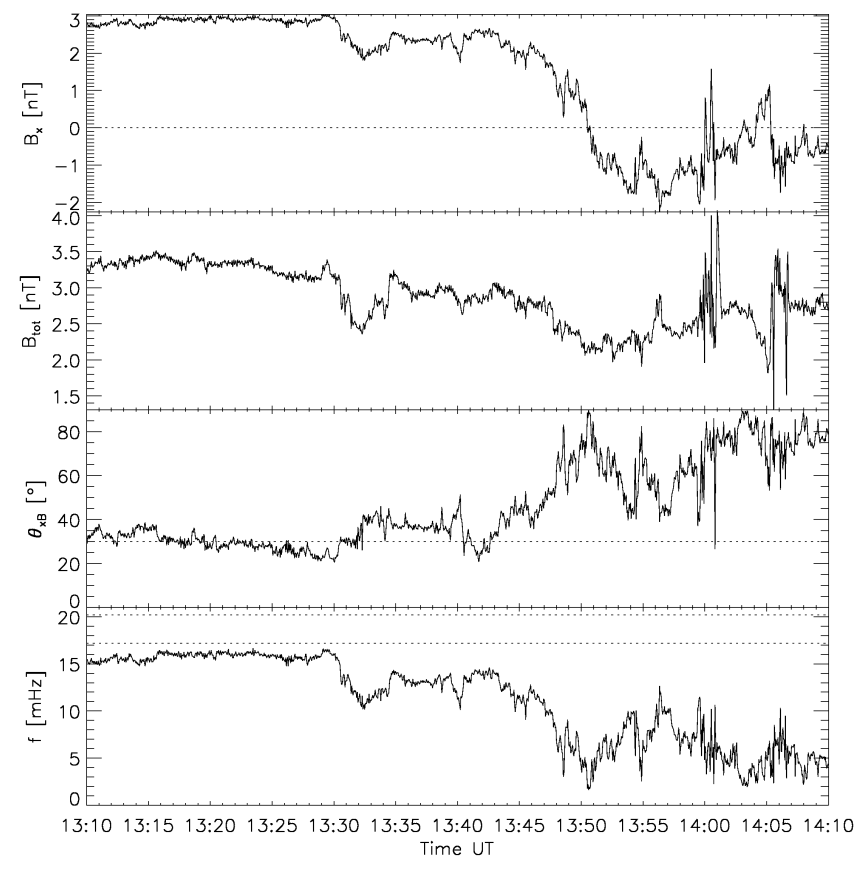

Fig. 8. Magnetic field data from GEOTAIL. The cone angle $\theta_{x B}$ and the predicted upstream generated frequency $f_{\text {pred }}$ are shown in the two bottom panels. The two horizontal dotted lines in the bottom panel show the observed frequencies.

are well reproduced by the model. Please bear in mind that the time resolution for the IMAGE magnetometers is $10 \mathrm{~s}$ such that the observed lags can only be determined to that accuracy.

All the above observations suggest an upstream source, hence the solar wind data has been analysed for possible triggers of the observed wave. Possible triggers for ULF waves in the magnetospheric cavity include increased shear flow at the magnetopause, generating waves through KelvinHelmholtz-Instabilities or solar wind buffeting due to oscillations or sudden changes in the solar wind dynamic pressure. The first option is unlikely, seeing that the maximum shear flow is achieved at the flanks of the magnetosphere. However, the onset time analysis suggests propagation of the wave essentially along the X GSM axis as opposed to originating from one of the flanks. The second option is ruled out since no oscillations in the solar wind dynamic pressure can be observed during the interval of interest.

Another wave generation mechanism is through instabilities between the solar wind plasma and ions backstreaming from the Earth's bow shock. Backstreaming ions are predominantly observed at a quasi-parallel shock, i.e. when the shock normal and the magnetic field vector are close to being (anti-)parallel. A generally used condition is that the angle between the two vectors is smaller than $30^{\circ}$. To our knowledge, two similar empirical models have been developed to estimate the frequency generated by this mechanism, one by
Takahashi et al. (1984) and one by Le and Russell (1996). Both models are parametrized by the IMF strength $B_{\text {tot }}$ in nT and the cone angle $\theta_{x B}$ as defined by

$\cos \left(\theta_{x B}\right)=\left|B_{x}\right| / B_{\text {tot }}$.

Here we use the more recent relation found by Le and Russell (1996) which is based on linear theory. It states that the predicted frequency $f_{\text {pred }}[\mathrm{mHz}]$ is dependent on $B_{\text {tot }}$ and $\theta_{x B}$ as

$f_{\text {pred }}[\mathrm{mHz}]=\left(0.72+4.67 \cos \left(\theta_{x B}\right)\right) B_{\text {tot }}$.

During the event, the GEOTAIL satellite was located inside the solar wind close to the Earth's bow shock on the evening side. From its magnetic field data $\theta_{x B}$ was calculated and the frequency of the upstream generated waves at the subsolar point has been estimated using the Eq. (6) (see Fig. 8). The predicted frequency matches reasonably well with the frequency observed, however Eq. (6) predicts slightly lower frequencies than those observed.

\subsection{Harmonic structure}

The cross-phase technique was employed (Kurchashov et al., 1987; Waters et al., 1991) to estimate the natural frequency of field lines. We can then investigate whether and how the observed oscillation was related to a normal mode of the considered field lines.

Under two assumptions one can estimate the natural (fundamental) frequency of a field line by calculating the crossphase spectrum of two adjacent stations at similar longitudes but different latitudes. Firstly it is assumed that quasirandom variations at the natural frequency of the field line are always present in the data. Secondly, the natural frequency of the poleward station is, mainly due to the longer field line, slightly lower than that of the equatorward station. The natural frequency of the field line located half way between the two stations is then that frequency, at which the cross-phase spectrum reaches its maximum. It has been shown (e.g. Waters et al., 1991) that this technique is reasonably robust in determining the natural frequency, even at low signal strengths.

The results for some exemplary SAMNET stations are shown in Fig. 9. The decreasing frequency with increasing latitude is nicely visible, as well as an expected variation of the fundamental frequency with local time (Waters et al., 1995). This technique yields clearer results for data from the evening flank. However, due to the symmetry of the CARISMA station and SAMNET station locations, conclusions drawn from the latter may be applicable to the morning sector (compare Fig. 5).

The fundamental frequencies $f_{\text {fund }}$ in $\mathrm{mHz}$ of 16 suitable pairs of stations from all three magnetometer arrays have been determined from the cross-phase spectra. Averaging all cross-phase values for each frequency bin over the time of the event yielded the natural frequency as that where the crossphase values were maximal. These frequencies are plotted over the average L-value of the station pairs in Fig. 10. 
The natural frequency decreases with increasing L-value, with a steeper gradient inside the plasmasphere than inside the magnetosphere. The position of the plasmapause can be identified as the position of a "bump" in the curve, as indicated by an arrow in Fig. 10. Its location coincides with the result derived from Eq. (4) as marked by the dotted vertical line. The results presented here are very similar to those presented in Fig. 3 of Menk et al. (2004). Least-square fitting a straight line to the data points in the two regimes as indicated by the solid lines in Fig. 10 yields two functional dependencies of the fundamental frequency $f_{\text {fund }}$ on L-value $L$ :

$\log _{10} f_{\text {fund }}(L)=\left\{\begin{array}{l}2.17-0.33 L, L \lesssim 4 \mathrm{R}_{\mathrm{e}} \\ 1.20-0.04 L, L \gtrsim 4 \mathrm{R}_{\mathrm{e}}\end{array}\right.$

During the fitting process the two points comprising the bump in Fig. 10 have been excluded. Using the above formula, an individual fundamental frequency for each station in Fig. 5 can be calculated.

It was already remarked that ground based magnetometer data show maximal amplitudes at two different latitudes. In an attempt to explain this amplitude distribution, the natural frequency of each station in Fig. 5 was plotted against the spectral power of each station's time series at $17.2 \mathrm{mHz}$.

The idea is that the amplitude of the pulsation and thus the spectral power at $17.2 \mathrm{mHz}$ of the ground based data was higher at latitudes, where the driving pulsation was harmonically related to the fundamental frequency because the driving wave could then have resonantly interacted with the local field line.

Panel a of Fig. 11 shows the spectral power at $17.2 \mathrm{mHz}$ over the fundamental frequency. The colours indicate the station's chain, blue for SAMNET stations, orange for IMAGE and green for CARISMA. The station abbreviations are given above or below their respective circle. The vertical bold dashed line marks the frequency of the driving pulsation, i.e. $17.2 \mathrm{mHz}$. The second vertical dashed line at $6.5 \mathrm{mHz}$ indicates the fundamental frequency of the field line whose second harmonic frequency matches the driving frequency.

The harmonic relationship for geomagnetic field line eigenfrequencies was studied for a dipole magnetic field by Schulz (1996). He found the frequency ratios at $L=6$ for the toroidal eigenmodes to be $f_{2} / f_{1}=2.63$ (compare Table 1 in Schulz, 1996). For different L-values the ratio varies by about 0.25 . In Fig. 11 this variation has been indicated by a gray band.

The lower panels of Fig. 11 show latitude profiles of the phase of selected stations determined by FFT analysis. Panel b shows a latitude profile of CARISMA stations and panel $c$ of SAMNET stations. The same stations as plotted in the lower panels are connected by lines in panel a.

The reader is reminded that the fundamental frequency of a field line is inversely proportional to the latitude of its foot point, i.e. the higher the latitude, the lower the fundamental frequency. Hence, Fig. 11 can also be looked at as a latitude

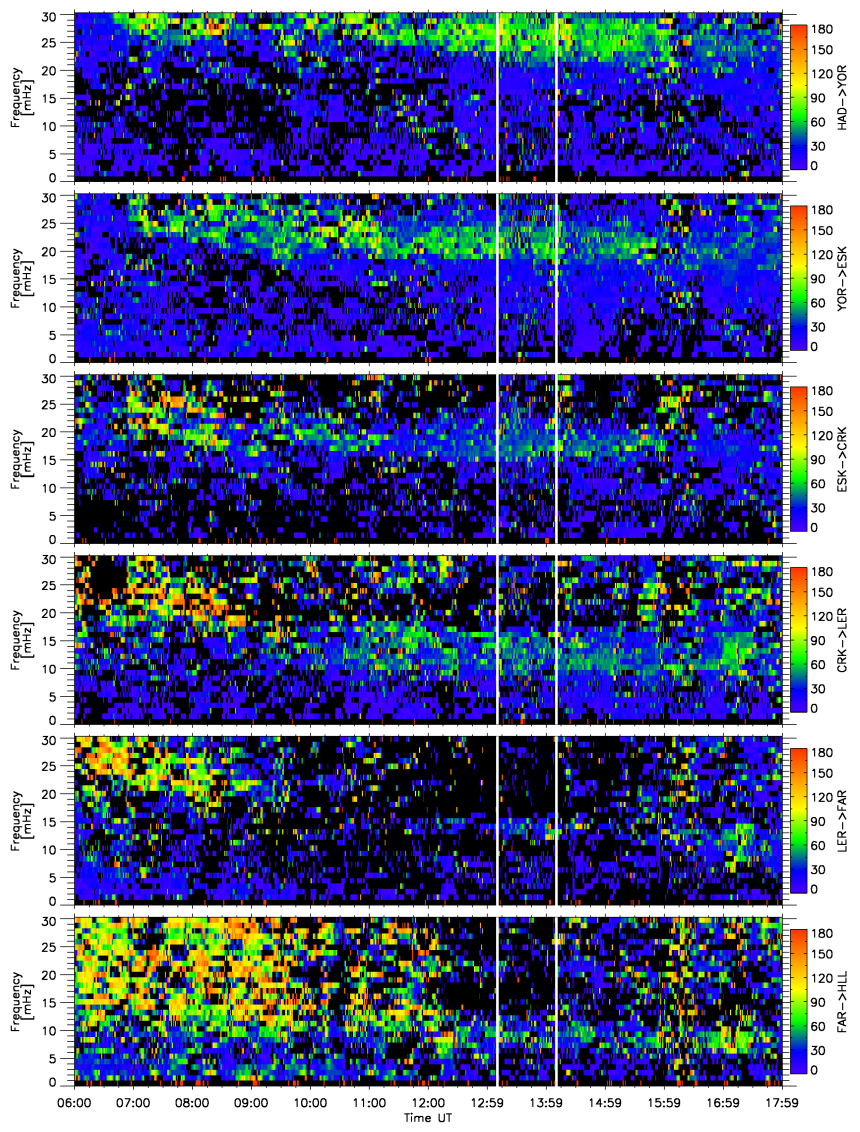

Fig. 9. Cross-phase spectra of stations belonging to the SAMNET array.

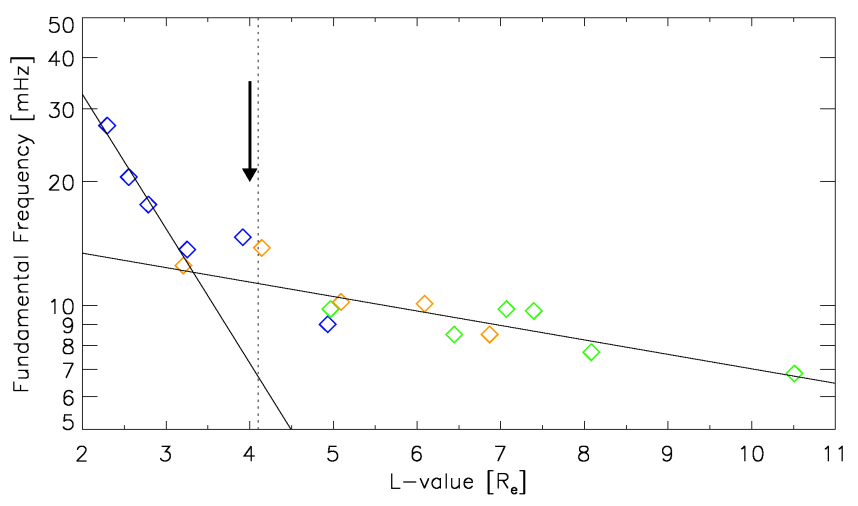

Fig. 10. Fundamental frequency over L-value. CARISMA stations are coloured green, SAMNET stations are blue and IMAGE stations are orange. The dotted vertical line indicates the position of the plasmapause predicted by Eq. (4).

profile of spectral power. For an ideal FLR such a profile shows a peak in power at the latitude of the resonant field line, accompanied by a $180^{\circ}$ phase shift across that latitude.

The orange circles representing the IMAGE data offer no clue as to whether resonant interaction between the global 

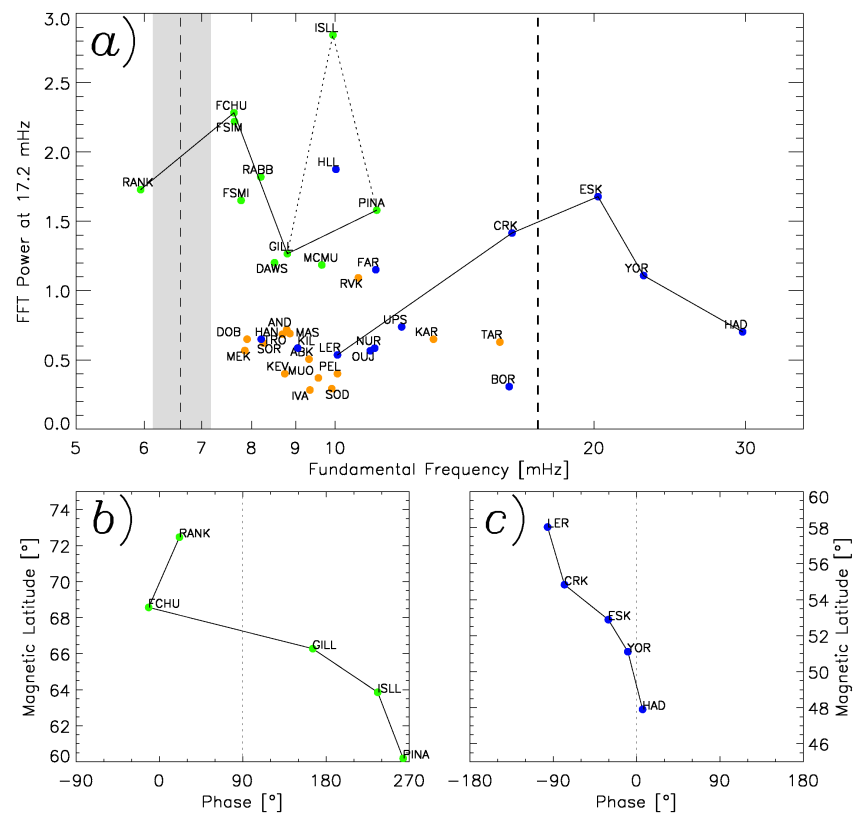

Fig. 11. (a) Fundamental frequency against the spectral power at 17.2 mHz. CARISMA stations are coloured green, SAMNET stations are blue and IMAGE stations are orange. (b) Latitude profile of the FFT phase of selected CARISMA stations; these are connected by straight lines in panel (a). (c) Latitude profile of the FFT phase of selected SAMNET stations; these are connected by straight lines in panel (a).

wave and the local field lines took place. This is due to the overall small signal strength at these stations combined with an unfortunate position of the fundamental frequencies between the two frequencies marked in Fig. 11.

The spectral powers of a latitude profile of stations belonging to the SAMNET array (HAD, YOR, ESK, CRK and LER) have been connected by straight lines in Fig. 11. The accompanying Fourier phases have been plotted in panel c. Both plots provide strong evidence that across this latitude ( $\sim 54^{\circ}$ magnetic latitude), where the driving and local fundamental frequency matched, resonant interaction took place and a FLR was generated.

The fundamental frequency at BOR was, according to Fig. 11, also close to that of the driving wave. Hence an enhancement in power could be expected. However, the power at BOR is low due to the station's position at 17:00 MLT where wave power is usually low. Conversely, power at HLL is high although its fundamental frequency is not close to any of the frequencies where resonant interaction is expected. Hence the amplitude distribution of the SAMNET data can only partly be explained by resonant interaction of the driving wave with a local field line.

A second latitude profile at higher latitudes on the dawn side consisting of CARISMA stations has also been marked in Fig. 11. It consists of the stations PINA, ISLL, GILL, FCHU, RANK. The corresponding Fourier phases are shown in panel $b$. Ignoring the power value at ISLL for a moment, again a clear signature of a FLR emerges. This indicates that the driving wave generated a FLR on the dawn side at a latitude where the second harmonic frequency of the local fundamental matched the driving frequency.

The above discussion shows that our idea of resonant interaction can only explain parts of the amplitude distribution of the Fourier powers with latitude. The high power values at ISLL and HLL remain puzzling because their fundamental frequency was not harmonically related to the driving frequency.

As can be seen in Fig. 2, the two Cluster satellites were located along the same bundle of field lines during the entire event. This, together with the global, simultaneous appearance of the wave in ground based magnetic data, strongly indicates that the observed wave packets were a temporal rather than a spatial feature. The separation of the two spacecraft was $2 \mathrm{R}_{\mathrm{e}}$ on the same field line, therefore phase information from the magnetic and electric field data can be used to learn more about the spatial structure of the wave along the field line.

The cross-phase method was used to determine the relative phase shift of the Fourier component at $17.2 \mathrm{mHz}$ and its evolution during the event for the field-aligned and azimuthal magnetic component (not shown) between the two spacecraft. The phase difference of the field-aligned component was constant at about $0^{\circ}$, whereas the phase shift in the azimuthal component was constant at $180^{\circ}$.

Because of the finite L-shell difference between Cluster 3 and 4, a measurable difference in resonance frequency at the two positions can be expected. Hence the relative phase between the signals of the two spacecraft would change with time if at their position a resonance would be observed. Since this is not the case, this confirms the observation that the Cluster satellites do not observe the signal of a resonating field line but that of an almost monochromatic driver.

Since the compressional parts of the magnetic field were in phase, the following considerations are only valid for the Alfvénic mode. The phase shift of the azimuthal magnetic components between the two spacecraft was $180^{\circ}$, whereas the radial electric components were in phase. Numerically solving the decoupled first-order wave equation along a dipole magnetic field line as derived in Sinha and Rajaram (1997) yields solutions for the magnetic and electric field. The following observations are qualitative and are only concerned with the node structure of the oscillation.

The density profile along the field line was assumed to be

$\rho=\rho_{0}\left(\frac{r_{0}}{r}\right)^{n}$

where $n$ is the density index and $\rho_{0}$ is the proton mass density at $r_{0}$, the cross-point of the field line considered, and $r$ is the geocentric distance to the position of interest on the field line. Denton et al. (2002) found the density index at an L-value of $5 \mathrm{R}_{\mathrm{e}}$, which is the position of the Cluster satellites, to be 

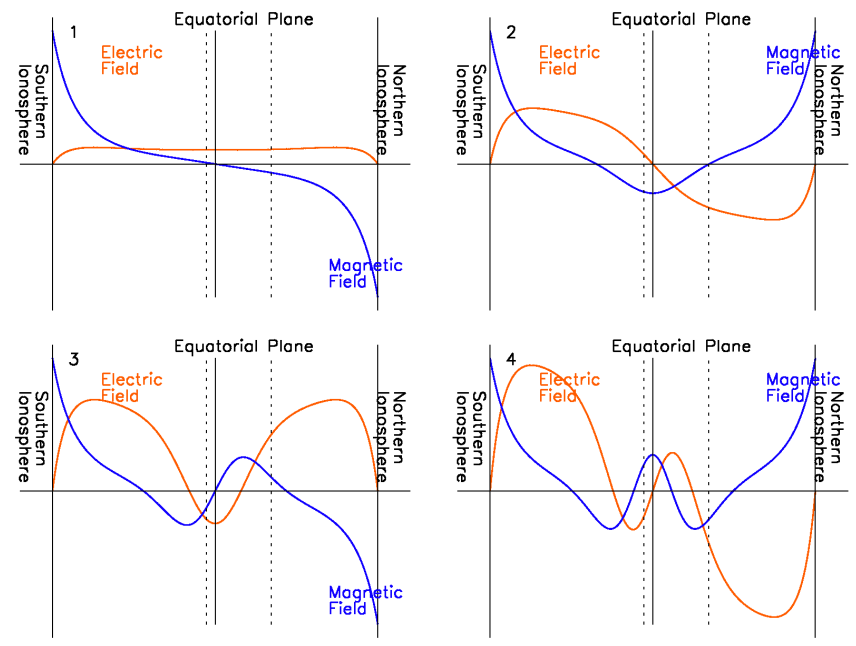

Fig. 12. Numerical solution of the wave equation on a dipolar field line. The first four harmonics are shown. Superimposed are the scaled positions of the Cluster 3 (right of the equatorial plane) and 4 (left) spacecraft.

around $n=1$. For calculations this value was chosen and it is worth noting that variations of the density index did not significantly change the positions of the nodes.

The result of a numerical integration using the standard fourth-order Runge-Kutta method is shown in Fig.. 12. Note that the delineation shows, for the sake of clarity, the two quantities at times of maximum amplitude. Superimposed are the positions of the Cluster spacecraft as dashed lines. Since the dipole field line in this sketch is represented by a straight line, the positions of the spacecraft have been scaled accordingly.

The numerical solution in Fig. 12 is based on the assumption that conductivities in both the northern and southern ionosphere are identical. This assumption does not hold for the position of the field lines adjacent to the Cluster satellites during the time of the event. The ionosphere at the southern foot point of the involved field lines was sun lit, whereas it was not at the northern end. Hence the ionospheric conductivities are expected to be lower at the northern than at the southern foot point. However, Streltsov and Lotko (1997) investigated the influence of finite ionospheric conductivity on the structure of dispersive, nonradiative FLRs for the first four odd harmonics. Their results are based on a linear, magnetically incompressible, reduced, two-fluid MHD model which includes effects of finite electron inertia (at low altitude) and finite electron pressure (at high altitude). They found that assuming different conductivities for the northern $\left(\Sigma_{P}=2 \mathrm{mho}\right)$ and southern $\left(\Sigma_{P}=\infty \mathrm{mho}\right)$ ionospheres do not significantly change the positions of the nodes.

The first and fourth harmonics seem possible solutions to explain the observed spatial phase shifts of the wave field, i.e. $0^{\circ}$ between the two radial electric and $180^{\circ}$ between the two azimuthal magnetic fields. Schäfer et al. (2007) found

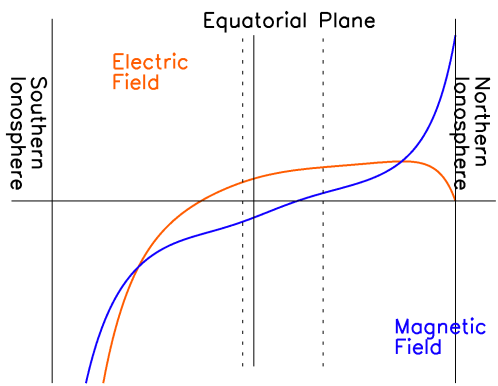

Fig. 13. Resulting wave structure when a $17.2 \mathrm{mHz}$ wave is fed into the Runge-Kutta algorithm. The vertical dashed lines again show the scaled positions of the Cluster spacecraft 3 (right of the equatorial plane) and 4 (left).

that the fundamental eigenfrequency of a toroidal standing wave on a dipole field line at $L=5 \mathrm{R}_{\mathrm{e}}$ under similar magnetospheric conditions is about $13 \mathrm{mHz}$. Similar values around $10 \mathrm{mHz}$ can be extracted by eye from the cross-phase spectra of data from the morning side ground based magnetometers. Hence it is more likely that the observed wave at $17.2 \mathrm{mHz}$ at the Cluster position has a node structure related to the fundamental or the second harmonic rather than one related to the fourth harmonic.

When feeding the Runge-Kutta algorithm with a $17.2 \mathrm{mHz}$ wave and assuming that the electric field had a node only on one end of the field line, the picture as delineated in Fig. 13 emerges. It has the same format as Fig. 12. Under the assumption that the electric field had a node in the northern hemisphere, the observed phase shifts in the Cluster data can be reproduced.

The astute reader will notice that the southern end of the field line connects to the sun-lit hemisphere. There the conductivities were probably higher than at the dark northern foot point. Hence the node in the electric field is more likely to have been in the southern end as opposed to the northern as delineated in Fig. 12. However, the observed phase shifts in the Cluster data can only be reproduced when assuming the node in the electric field to have been at the northern foot point. It is possible that auroral precipitation changed the local ionospheric conductivities at the foot print of the field lines connected to the satellites such that the above scenario becomes plausible.

On 7 November 2005 five wave packets in the Pc4 frequency range have been observed in ground based magnetometer data from stations spanning virtually all latitudes and all MLTs. The most prominent of the wave packets had a frequency of $17.2 \mathrm{mHz}$ and an azimuthal wave number $m$ of about 1 . The frequency was constant at all latitudes.

The onset times of the pulsation and the Poynting flux calculated from space based data show that the wave was propagating from the dayside into the nightside. This suggests an upstream source. Since no apparent trigger was found in solar wind data, we believe that the wave was generated 
by backstreaming ions at the Earth's bow shock. The compressional part of this wave then propagated through the bow shock and the magnetosheath into the magnetosphere. There its energy partially converted into Alfvénic modes which were observed as a global phenomenon by ground instruments. Space based instruments registered both modes.

Due to the unique spatial arrangement of two Cluster spacecraft the harmonic structure of the wave packet along the same bundle of field lines has been studied. A simple comparison between the phase shifts of the azimuthal magnetic and radial electric field between the two spacecraft and numerical solutions of the wave field suggests that at an Lvalue of $5 \mathrm{R}_{\mathrm{e}}$ on the dawn flank the observed pulsation had a node structure similar to the fundamental of the adjacent field line. However uncertainties in the numerical solution exist that could effect the node structure and hence its interpretation.

Using the cross-phase technique, fundamental frequencies of field lines adjacent to ground based magnetometers have been calculated and compared with that of the observed pulsation and the Cluster observations. At latitudes comparable with that of the Cluster foot print these data suggest that the pulsation was oscillating at a frequency between the local fundamental and second harmonic frequency. This observation is supported by the observed phase shift at the Cluster satellites and numerical simulations.

On the dusk side the incident wave generated a FLR at a latitude where the local fundamental frequency matched the driving frequency. On the dawn side indications for a FLR are found across the latitude where the driving frequency matched the second harmonic of the local fundamental.

Some of the features of the discussed event remain puzzling. The high amplitudes observed on the ground at HLL and ISLL cannot be explained by resonant interaction of local field lines and the driving wave (see Fig. 11). The splitting of the wave packet as observed on the ground could be due to different damping rates at different latitudes (see Fig. 1). Lower damping at lower latitudes could be responsible for the merging of two wave packets into what appears to be one. And lastly the signal at double the main frequency in the spacecraft data (see Fig. 3 and 4) could be connected to mechanisms described in Takahashi and McPherron (1982), Higuchi et al. (1986) and Mann et al. (1999).

Acknowledgements. L. Clausen acknowledges funding from the European Commission under the Marie Curie Host Fellowship for Early Stage Research Training SPARTAN, Contract No MEST-CT2004-007512, University of Leicester, UK.

The authors would like to thank the following people and institutions for providing data and maintaining instruments: I. R. Mann at the University of Alberta and the CARISMA team, CARISMA is operated by the University of Alberta, funded by the Canadian Space Agency; A. Viljanen at the Finnish Meteorological Institute and the IMAGE team; F. Honary at Lancaster University and the SAMNET team, SAMNET is a PPARC National Facility operated by Lancaster University; A. Balogh at Imperial Col- lege and the Cluster/FGM team; M. Andre at the Swedish Institute of Space Physics and the Cluster/EFW team; the Cluster Active Archive team; T. Nagai at the Tokyo Institute of Technology and the GEOTAIL/MGF team. GEOTAIL/MGF data was provided through DARTS at the Institute of Space and Astronautical Science (ISAS) in Japan.

The authors would also like to thank C. Waters of the University of Newcastle, Australia, for providing code for calculating cross-phase spectra.

Topical Editor I. A. Daglis thanks K. Takahashi and M. Vellante for their help in evaluating this paper.

\section{References}

Baddeley, L. J., Yeoman, T. K., Wright, D. M., Davies, J. A., Trattner, K. J., and Roeder, J. L.: Morning sector drift-bounce resonance driven ULF waves observed in artificially-induced HF radar backscatter, Ann. Geophys., 20, 1487-1498, 2002.

Balogh, A., Carr, C. M., Acuña, M. H., Dunlop, M. W., Beek, T. J., Brown, P., Fornaçon, K.-H., Georgescu, E., Glassmeier, K. H., Harris, J., Musmann, G., Oddy, T., and Schwingenschuh, K.: The Cluster Magnetic Field Investigation: Overview of In-Flight Performance and Initial Results, Ann. Geophys., 19, 1207-1217, 2001.

Barnes, A.: A theory of generation of bow-shock-associated hydromagnetic waves in the upstream interplanetary medium, Cosmic Electrodyn., 1, 90-+, 1970.

Chen, L. and Hasegawa, A.: A theory of long-period magnetic pulsations, 1. Steady state excitation of field line resonance, J. Geophys. Res., 79, 1024-1032, 1974.

Chi, P. J. and Russell, C. T.: Phase skipping and Poynting flux of continuous pulsations, J. Geophys. Res., 103, 29479-29492, doi:10.1029/98JA02101, 1998.

Chi, P. J., Russell, C. T., Raeder, J., Zesta, E., Yumoto, K., Kawano, H., Kitamura, K., Petrinec, S. M., Angelopoulos, V., Le, G., and Moldwin, M. B.: Propagation of the preliminary reverse impulse of sudden commencements to low latitudes, J. Geophys. Res., 106, 18 857-18 864, doi:10.1029/2001JA900071, 2001.

Denton, R. E., Goldstein, J., Menietti, J. D., and Young, S. L.: Magnetospheric electron density model inferred from Polar plasma wave data, J. Geophys. Res., 107, 25-1, doi:10.1029/ 2001JA009136, 2002.

Escoubet, C. P., Fehringer, M., and Goldstein, M.: The Cluster Mission, Ann. Geophys., 19, 1197-1200, 2001.

Greenstadt, E. W., Olson, J. V., Loewen, P. D., Singer, H. J., and Russell, C. T.: Correlation of PC 3, 4, and 5 activity with solar wind speed, J. Geophys. Res., 84, 6694-6696, 1979.

Gustafsson, G., Bostrom, R., Holback, B., Holmgren, G., Lundgren, A., Stasiewicz, K., Ahlen, L., Mozer, F. S., Pankow, D., Harvey, P., Berg, P., Ulrich, R., Pedersen, A., Schmidt, R., Butler, A., Fransen, A. W. C., Klinge, D., Thomsen, M., Falthammar, C.G., Lindqvist, P.-A., Christenson, S., Holtet, J., Lybekk, B., Sten, T. A., Tanskanen, P., Lappalainen, K., and Wygant, J.: The Electric Field and Wave Experiment for the Cluster Mission, Space Sci. Rev., 79, 137-156, 1997.

Higuchi, T., Kokubun, S., and Ohtani, S.: Harmonic structure of compressional Pc5 pulsations at synchronous orbit, Geophys. Res. Lett., 13, 1101-1104, 1986. 
Krauss-Varban, D.: Bow Shock and Magnetosheath Simulations: Wave Transport and Kinetic Properties, in: Solar Wind Sources of Magnetospheric Ultra-Low-Frequency Waves, edited by: Engebretson, M. J., Takahashi, K., and Scholer, M., 121-134, 1994.

Kurchashov, I. P., Nikomarov, I. S., Pilipenko, V. A., and Best, A.: Field line resonance effects in local meridional structure of midlatitude geomagnetic pulsations, Ann. Geophys., 5, 147-154, 1987.

Larsen, B. A., Klumpar, D. M., and Gurgiolo, C.: Correlation between plasmapause position and solar wind parameters, J. Atmos. Terr. Phys., 69, 334-340, doi:10.1016/j.jastp.2006.06.017, 2007.

Le, G. and Russell, C. T.: Solar wind control of upstream wave frequency, J. Geophys. Res., 101, 2571-2576, doi:10.1029/ 95JA03151, 1996.

Le, G., Chi, P. J., Goedecke, W., Russell, C. T., Szabo, A., Petrinec, S. M., Angelopoulos, V., Reeves, G. D., and Chun, F. K.: Magnetosphere on May 11, 1999, the Day the Solar Wind Almost Disappeared, II Magnetic Pulsations in Space and on the Ground, Geophys. Res. Lett., 27, 2165-+, 2000.

Mann, I. R., Chisham, G., and Wanliss, J. A.: Frequency-doubled density perturbations driven by ULF pulsations, J. Geophys. Res., 104, 4559-4566, doi:10.1029/1998JA900136, 1999.

Menk, F. W., Mann, I. R., Smith, A. J., Waters, C. L., Clilverd, M. A., and Milling, D. K.: Monitoring the plasmapause using geomagnetic field line resonances, J. Geophys. Res., 109, 4216+, doi:10.1029/2003JA010097, 2004.

Nosé, M.: ULF pulsations observed by the ETS-VI satellite: Substorm associated azimuthal $\mathrm{Pc} 4$ pulsations on the nightside, Earth Planets Space, 50, 63-80, 1998.

Odera, T. J., van Swol, D., Russell, C. T., and Green, C. A.: PC 3,4 magnetic pulsations observed simultaneously in the magnetosphere and at multiple ground stations, Geophys. Res. Lett., 18, 1671-1674, 1991.

Samson, J. C. and Rostoker, G.: Latitude-dependent characteristics of high-latitude Pc 4 and Pc 5 micropulsations, J. Geophys. Res., 77, 6133-6144, 1972.

Samson, J. C., Harrold, B. G., Ruohoniemi, J. M., Greenwald, R. A., and Walker, A. D. M.: Field line resonances associated with MHD waveguides in the magnetosphere, Geophys. Res. Lett., 19, 441-444, 1992.

Schäfer, S., Glassmeier, K. H., Eriksson, P. T. I., Pierrard, V., Fornaçon, K. H., and Blomberg, L. G.: Spatial and temporal characteristics of poloidal waves in the terrestrial plasmasphere: a CLUSTER case study, Ann. Geophys., 25, 1011-1024, 2007.
Schulz, M.: Eigenfrequencies of geomagnetic field lines and implications for plasma-density modeling, J. Geophys. Res., 101, 17385-17398, doi:10.1029/95JA03727, 1996.

Sentman, D. D., Edmiston, J. P., and Frank, L. A.: Instabilities of low frequency, parallel propagating electromagnetic waves in the earth's foreshock region, J. Geophys. Res., 86, 7487-7497, 1981.

Shue, J.-H., Chao, J. K., Fu, H. C., Russell, C. T., Song, P., Khurana, K. K., and Singer, H. J.: A new functional form to study the solar wind control of the magnetopause size and shape, J. Geophys. Res., 102, 9497-9512, doi:10.1029/97JA00196, 1997.

Sinha, A. K. and Rajaram, R.: An analytic approach to toroidal eigen mode, J. Geophys. Res., 102, 17 649-17 657, doi:10.1029/ 97JA01039, 1997.

Southwood, D. J.: Some features of field line resonances in the magnetosphere, Planet. Space Sci., 22, 483-491, 1974.

Streltsov, A. and Lotko, W.: Influence of the finite ionospheric conductivity on dispersive, nonradiative field line resonances, Ann. Geophys., 15, 625-633, 1997.

Takahashi, K. and McPherron, R. L.: Harmonic structure of Pc 3-4 pulsations, J. Geophys. Res., 87, 1504-1516, 1982.

Takahashi, K., McPherron, R. L., and Terasawa, T.: Dependence of the spectrum of Pc 3-4 pulsations on the interplanetary magnetic field, J. Geophys. Res., 89, 2770-2780, 1984.

Tamao, T.: The Structure of Three-dimensional Hydromagnetic Waves in a Uniform Cold Plasma, J. Geomagn. Geoelectr., 16, 89-114, 1964.

Troitskaya, V. A., Plyasova-Bakunina, T. A., and Gul'elmi, A. V.: Relationship between Pc 2-4 pulsations and the interplanetary field, Dokl. Akad. Nauk. SSSR, 197, 1313, 1971.

Tsyganenko, N. A.: Modeling the Earth's magnetospheric magnetic field confined within a realistic magnetopause, J. Geophys. Res., 100, 5599-5612, 1995.

Walker, A. D. M., Greenwald, R. A., Stuart, W. F., and Green, C. A.: STARE auroral radar observations of Pc 5 geomagnetic pulsations, J. Geophys. Res., 84, 3373-3388, 1979.

Waters, C. L., Menk, F. W., and Fraser, B. J.: The resonance structure of low latitude Pc3 geomagnetic pulsations, Geophys. Res. Lett., 18, 2293-2296, 1991.

Waters, C. L., Samson, J. C., and Donovan, E. F.: The temporal variation of the frequency of high latitude field line resonances, J. Geophys. Res., 100, 7987-7996, 1995. 